\title{
Inactivation of Tsc2 in Mesoderm-Derived Cells Causes Polycystic Kidney Lesions and Impairs Lung Alveolarization
}

Siying Ren, ${ }^{\dagger \dagger}$ Yongfeng Luo, ${ }^{\dagger}$ Hui Chen, ${ }^{\dagger}$ David Warburton, ${ }^{\dagger \dagger}$ Hilaire C. Lam, ${ }^{\S}$ Larry L. Wang, ${ }^{\dagger}$ Ping Chen, ${ }^{*}$ Elizabeth P. Henske, and Wei Shi ${ }^{\dagger+}$

From the Department of Respiratory Medicine,* The Second Xiangya Hospital, Central-South University, Changsha, People's Republic of China; the Developmental Biology and Regenerative Medicine Program, ${ }^{\dagger}$ The Saban Research Institute, Children's Hospital Los Angeles, Los Angeles, California; the Department of Surgery, ${ }^{\ddagger}$ Keck School of Medicine, University of Southern California, Los Angeles, California; and the Division of Pulmonary and Critical Care Medicine,,$^{\S}$ Brigham and Women's Hospital and Harvard Medical School, Boston, Massachusetts

\author{
Accepted for publication \\ August 29, 2016. \\ Address correspondence to Wei \\ Shi, M.D., Ph.D., Children's \\ Hospital Los Angeles, 4650 \\ Sunset Blvd, MS 35, Los \\ Angeles, CA 90027; or Eliz- \\ abeth P. Henske, M.D., Thorn \\ 826, Brigham and Women's \\ Hospital, 45 Francis Street, \\ Boston, MA 02115. E-mail: \\ wshi@chla.usc.edu or \\ ehenske@bwh.harvard.edu.
}

\begin{abstract}
The tuberous sclerosis complex (TSC) proteins are critical negative regulators of the mammalian/ mechanistic target of rapamycin complex 1 pathway. Germline mutations of TSC1 or TSC2 cause TSC, affecting multiple organs, including the kidney and lung, and causing substantial morbidity and mortality. The mechanisms of organ-specific disease in TSC remain incompletely understood, and the impact of TSC inactivation on mesenchymal lineage cells has not been specifically studied. We deleted Tsc2 specifically in mesoderm-derived mesenchymal cells of multiple organs in mice using the Dermo1Cre driver. The Dermo1-Cre-driven Tsc2 conditional knockout mice had body growth retardation and died approximately 3 weeks after birth. Significant phenotypes were observed in the postnatal kidney and lung. Inactivation of Tsc2 in kidney mesenchyme caused polycystic lesions starting from the second week of age, with increased cell proliferation, tubular epithelial hyperplasia, and epithelialmesenchymal transition. In contrast, Tsc2 deletion in lung mesenchyme led to decreased cell proliferation, reduced postnatal alveolarization, and decreased differentiation with reduced numbers of alveolar myofibroblast and type II alveolar epithelial cells. Two major findings thus result from this model: inactivation of Tsc2 in mesoderm-derived cells causes increased cell proliferation in the kidneys but reduced proliferation in the lungs, and inactivation of Tsc2 in mesoderm-derived cells causes epithelial-lined renal cysts. Therefore, Tsc2-mTOR signaling in mesenchyme is essential for the maintenance of renal structure and for lung alveolarization. (Am J Pathol 2016, 186: 3261-3272; http:// dx.doi.org/10.1016/j.ajpath.2016.08.013)
\end{abstract}

Tuberous sclerosis complex (TSC) is an autosomal dominant genetic disorder resulting from germline loss-offunction mutations in the TSC1 or TSC 2 gene. ${ }^{1,2}$ The TSC1/TSC2 protein complex has GTPase-activating protein activity toward Rheb, an upstream regulator of mTOR, and thus negatively regulates mammalian/mechanistic target of rapamycin complex 1 (mTORC1) signaling activity by converting active Rheb-GTP to inactive Rheb-GDP. Therefore, abrogation of TSC1 or TSC2 causes dysregulation of mTORC1-dependent pathways, including protein translation, pyrimidine and purine biosynthesis, and autophagy. In addition to neurological disease, which includes seizures and autism, TSC patients have clinical manifestations in multiple organs, including the kidney, heart, lung, and skin. In the kidney, a variety of lesions, such as angiomyolipoma, isolated or multiple renal cysts, and renal cell carcinoma, can occur in TSC patients. In the lung, TSC

Supported by NIH grants HL068597 (W.S.), HL118760 (E.P.H.), and DK096556 (E.P.H.); a Tuberous Sclerosis Alliance Research grant (W.S.); the Hunan Province Health Department Research Fund B2014-020 (S.R.); the Hunan Province Natural Science Foundation of China 14JJ7012 (S.R.); LAM Foundation Postdoctoral Fellowship (H.C.L); and the Engles Program in TSC and LAM Research (E.P.H.).

Disclosures: None declared. 
patients can develop multifocal micronodular pneumocyte hyperplasia and lymphangioleiomyomatosis (LAM). ${ }^{3,4}$ LAM is a progressive disease affecting almost exclusively women and resulting in cystic lung degeneration. Up to $80 \%$ of women with TSC have multiple lung cysts by the age of 40 years, although only a minority will develop symptomatic LAM. Men with TSC can also develop cystic lung disease, although it is almost always asymptomatic. LAM also occurs in a sporadic form in women who do not have TSC. Sporadic LAM is associated with somatic inactivation of the TSC1 or TSC2 gene. ${ }^{5,6}$ The pathogenic mechanisms underlying the development of the clinical manifestations of TSC, in particular the pathogenesis of LAM, are not completely understood. One major challenge is the limitation of existing TSC disease models because conventional homozygous $T s c 1$ or $T s c 2$ knockout mice are embryonic lethal, and heterozygous $T s c 1$ or $T s c 2$ knockout mice develop epithelial renal lesions and tumors as their primary phenotypes. $^{7-9}$ This is a striking contrast to human TSC, in which mesenchymal lesions of the kidney (angiomyolipomas) and lung (LAM) are major causes of morbidity and mortality. It is still unclear how the TSC protein complex functions in different cell compartments, such as epithelial cells versus mesenchymal cells, during organ development and during post-developmental tissue homeostasis. Although genetic deletions of $T s c 1$ or $T s c 2$ in distinct cell lineages, such as neurons, neural crest-derived cells, endothelia, cardiomyocytes, and differentiated smooth muscle cells, have been reported in mice, ${ }^{10-14}$ the consequences of Tsc1 or Tsc2 inactivation in pan-mesenchymal cells has remained unknown.

We have investigated the specific function of Tsc2 in mesoderm-derived cells from the beginning of development in vivo. We achieved this by crossing a mesoderm-specific Cre-expressing mouse line Dermol-Cre with floxed-Tsc2 mice to generate mesenchyme-specific $T s c 2$ conditional knockout mice. ${ }^{15,16}$ Although these $T s c 2$ conditional knockout mice were alive postnatally, postnatal growth retardation, polycystic kidney pathology, and defective lung alveolarization were detected during postnatal development. These mice died approximately 3 weeks after birth, consistent with an indispensible role of Tsc2 in mesenchymal cells in regulating both organ development and tissue homeostasis.

\section{Materials and Methods}

\section{Mouse Strains and Breeding}

Mesenchyme-specific Dermol-Cre mice were gifts from Dr. David Ornitz (Washington University, St. Louis, MO). ${ }^{15}$ Floxed-Tsc2 $\left(T s c 2^{f x / f x}\right)$ mice were gifts from Dr. Michael Gambello's laboratory (Emory University, Atlanta, GA). ${ }^{16}$ $m T-m G$ double fluorescence reporter mice and $G t(R O S A)$ 26 Sor ${ }^{\mathrm{tm} 1 \mathrm{Sor}}$ LacZ reporter mice were obtained from Jackson Laboratory (Bar Harbor, ME). ${ }^{17,18}$ All mice were bred in C57BL/6 stain background, and housed in pathogen-free conditions at the animal facility of Children's Hospital Los Angeles. The procedures for mouse breeding and tissue harvest were approved by the Institutional Animal Care and Use Committee at the Saban Research Institute of Children's Hospital Los Angeles. Mouse genotypes were determined by genomic DNA PCR using tail biopsy samples. The genotyping PCR primers were described in previous publications. ${ }^{16,19}$

\section{Histology and Morphometric Analysis}

Fresh tissues at different ages were harvested from euthanized mice, and examined under dissecting microscope. Samples were then either fixed in $4 \%$ buffered paraformaldehyde or frozen in liquid nitrogen. The fixed tissues were dehydrated and embedded in paraffin, and cut into sections ( $5 \mu \mathrm{m}$ thick). The tissue sections were stained with hematoxylin and eosin or periodic acid-Schiff (Sigma Aldrich, St. Louis, MO). Lung elastin was stained using Hart's resorcin-fuchsin method, as described in our previous publication. ${ }^{19}$ For lung morphometric analysis, five sections of each tissue were randomly chosen at approximately $200-\mu \mathrm{m}$ intervals and stained with hematoxylin and eosin. The mean linear intercept was used to measure average size of alveoli, as described previously. ${ }^{19}$ Results were analyzed with unpaired $t$-tests to compare the differences between mean values, and considered significant if $P<0.05$. To avoid the sex differences in organ maturation, this quantitative comparison was also performed among fetuses with different sex at each time point.

\section{Immunostaining and Detection}

Tissue sections were deparaffinized and hydrated, and antigen recovery was performed by boiling tissue slides in Tris-EDTA buffer ( $\mathrm{pH}$ 9.0) for 30 minutes. The tissue sections were blocked in 5\% donkey normal serum and $0.5 \%$ Triton X-100 for 1 hour, and then incubated with the following primary antibodies overnight at $4^{\circ} \mathrm{C}$ : mouse anti-green fluorescent protein (GTX628528; GeneTex, Irvine, CA); goat anti-platelet endothelial cell adhesion molecule 1 (sc-1506), goat anti-CC10 (sc-9772), and goat anti-Sp-C (sc-7706; Santa Cruz Biotechnology, Santa Cruz, CA); rabbit anti-red fluorescent protein for Tomato protein (600-401-379; Rockland, Limerick, PA); rat antiCdh1 (13-1900; ThermoFisher, Waltham, MA), mouse anti- $\beta$-tubulin IV (MU178-UC; BioGenex, Fremont, CA); fluorescein-Lotus tetragonobolus lectin and rhodamineDolichos biflorus agglutinin (FL-1321 and RL-1032; Vector Laboratories, Burlingame, CA); mouse anti-cytokeratin and mouse anti- $\alpha$-smooth muscle actin (C2562 and A2547; Sigma Aldrich); rabbit anti-phospho-S6 (Ser235/236) and rabbit anti-vimentin (4858 and 5741; Cell Signaling Technology, Danvers, MA); and hamster anti-T1 $\alpha$ (DSHB at the University of Iowa, Iowa City). Alexa Fluro 488-, Alexa Fluro 594-, or Alexa Fluro 647-conjugated donkey secondary antibodies (ThermoFisher) were used, and cell 
nuclei were counterstained by DAPI in the mounting medium (Vector Laboratories). Fluorescence images were taken using the Zeiss LSM710 confocal microscope (Carl Zeiss, Oberkochen, Germany) at the Imaging Core Facility of Children's Hospital Los Angeles. Active caspase 3 staining was performed by immunohistochemistry using rabbit anti-cleaved caspase 3 antibody (9661; Cell Signaling Technology) and second anti-rabbit antibody-horseradish peroxidase conjugate, followed by color development with 3,3'-diaminobenzidine and hematoxylin counterstaining.

\section{Western Blot}

Detection of proteins in tissue lysate by Western blot has been previously described with the following minor modification. ${ }^{20}$ Equal amounts $(50 \mu \mathrm{g})$ of total tissue lysate proteins were separated in mini tris-glycine extended precast gels ( $4 \%$ to $15 \%$ gradient; Bio-Rad, Hercules, CA) and transferred into polyvinylidene difluoride membrane using Bio-Rad's TransBlot Turbo Transfer System. Proteins of interest were detected using the following specific antibodies: rabbit anti-Tsc2 (GTX61245; GeneTex); rabbit anti-phospho-S6 (Ser235/ 236) and mouse anti-S6 (4858 and 2317; Cell Signaling Technology); and rabbit anti- $\beta$-actin (G046; Applied Biological Materials, Richmond, BC, Canada).

\section{Cell Proliferation}

To detect proliferating cells in vivo, cell nuclear $5^{\prime}$-ethynyl$2^{\prime}$-deoxyuridine (EdU) labeling was used. ${ }^{21}$ Briefly, mice were i.p. injected with EdU $(5 \mathrm{mg} / \mathrm{kg}$ body weight; ThermoFisher) 3 hours before harvesting tissue samples. EdU is a thymidine analogue that is incorporated into newly synthesized chromosome DNA. Incorporated EdU was detected using Alexa Fluor azide (Life Technologies), and cell nuclei were counterstained with DAPI. The image analysis was performed in four random fields per slide from a total of four slides per mouse by an experienced observer (Y.L.) blind to the mouse genotype. The ratios of EdU-labeled nuclei/total DAPI-stained nuclei were used to evaluate cell proliferation, and the experiments were repeated in more than three mice within each genotype group.

\section{Serum Cystatin C Measurement}

Blood was collected from five $T s c 2$ conditional knockout (CKO) and wild-type (WT) littermate control pairs around postnatal day 20 (P20). The sera were diluted 200-fold, and used for cystatin $\mathrm{C}$ measurement using a commerical enzyme-linked immunosorbent assay kit (ThermoFisher). Paired sample $t$-test was used for the analysis.

\section{Statistical Analysis}

All experiments were repeated at least three times. The quantitative data were presented as means \pm SD. Statistical analyses were performed using $t$-tests, with $P \leq 0.05$ considered significant.

\section{Results}

Conditional Deletion of Tsc2 in Mesoderm-Derived Cells Results in Postnatal Growth Retardation and Lethality

It has been reported that Dermol promoter-driven Cre expression is specific for certain lineages of mesoderm-derived cells in a variety of organs in the Dermol-Cre knockin fetal mice, ${ }^{15}$ and we verified this Cre expression pattern in embryonic day 14.5 Dermol-Cre fetuses using Gt(ROSA)26Sor ${ }^{\mathrm{tm} 1 \text { Sor }}$ LacZ reporter mice (Figure 1A). We crossed Dermol-Cre with floxed-Tsc2 mice $\left(T s c 2^{\mathrm{fx} / \mathrm{fx}}\right)$ to specifically delete $T s c 2$ gene in Dermo1-expressing lineages of mesenchymal cells within multiple organs/systems during development. The mice were born with the expected mendelian ratio of genotypes and sexes. All control pups had normal development and weight gain with normal survival through 12 months of age. Dermol-Crel $T s c 2^{\mathrm{fx} / \mathrm{fx}}$ conditional knockout (abbreviated as $T s c 2$ CKO) mice were born with notably smaller body size and lower body weight than their littermate controls. These differences became more obvious during postnatal growth (Figure 1, B and C), suggesting significant postnatal growth retardation in $T s c 2$ CKO mice. Heterozygous Tsc2 CKO (Dermol-Cre/Tsc $2^{\mathrm{fx} /+}$ ) mice had body weight and size comparable to their WT littermates, and did not develop noticeable phenotypic abnormalities up to 5 months of age (data not shown).

The homozygous $T s c 2$ CKO mice died approximately 3 weeks after birth, with median survival of P20 (Figure 1D). No sex difference was observed for Tsc2 CKO mouse survival. Unlike the phenotypic changes seen in liver, cardiac, and central neuronal systems of germline $T s c 2$ mutant animals, ${ }^{8,22,23}$ there were no histologically obvious abnormalities in brain, liver, or heart in the Tsc2 CKO mice at P14 (Figure 2). In contrast, the kidney and lung were clearly abnormal at this age (see Results below for detail). The potential causes for the lethality in $T s c 2$ CKO mice are further analyzed and discussed below.

\section{Mesodermal Tsc2 Abrogation Results in Severe Polycystic Kidney Lesions}

Dermo1-Cre-driven gene deletion, shown by green fluorescent protein expression in a mT-mG reporter line, ${ }^{17}$ occurred in renal mesenchymal cells of both the cortical and medullary regions, but not in tubular epithelial cells or endothelial cells, shown by $\mathrm{Cdh}^{+}\left(\mathrm{E}-\mathrm{cadherin}{ }^{+}\right)$and platelet endothelial cell adhesion molecule $1^{+}$staining, respectively (Figure 3, A and B). Reduction of Tsc2 protein and activation of the mTORC1 pathway (increased phosphorylation of ribosomal protein S6) were verified by Western blotting of the entire Tsc 2 CKO kidney tissue lysate (Figure 3C). Examination of the moribund $T s c 2$ CKO mice sacrificed at approximately 3 weeks after birth revealed obvious changes in kidney size and appearance, which 
A

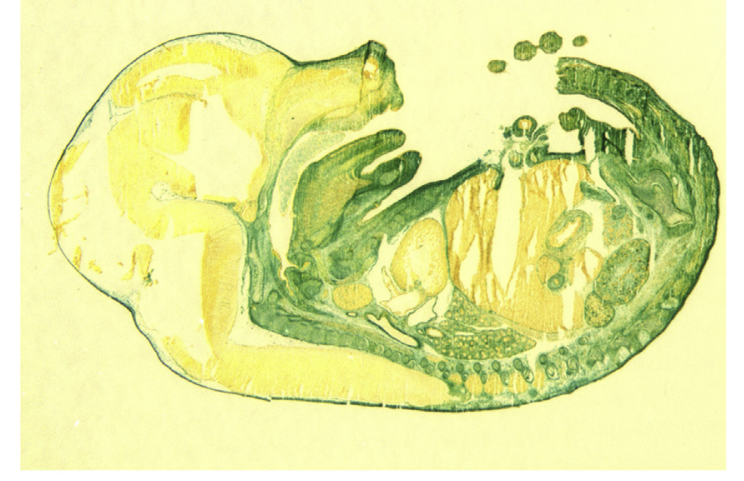

B
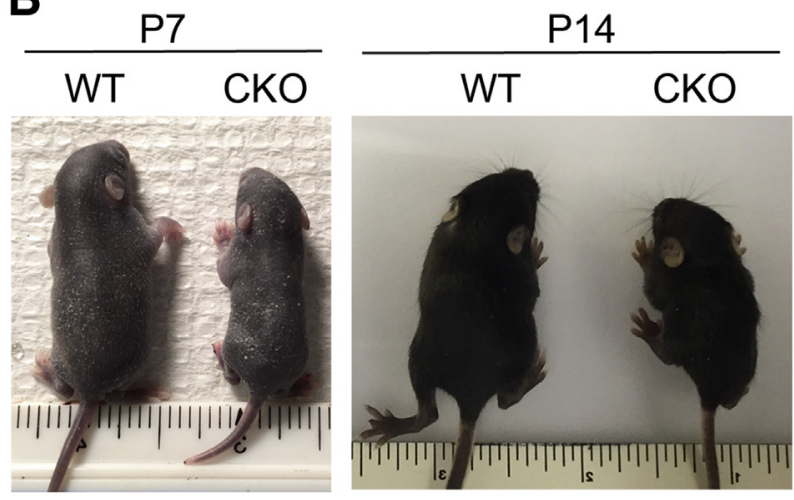

C
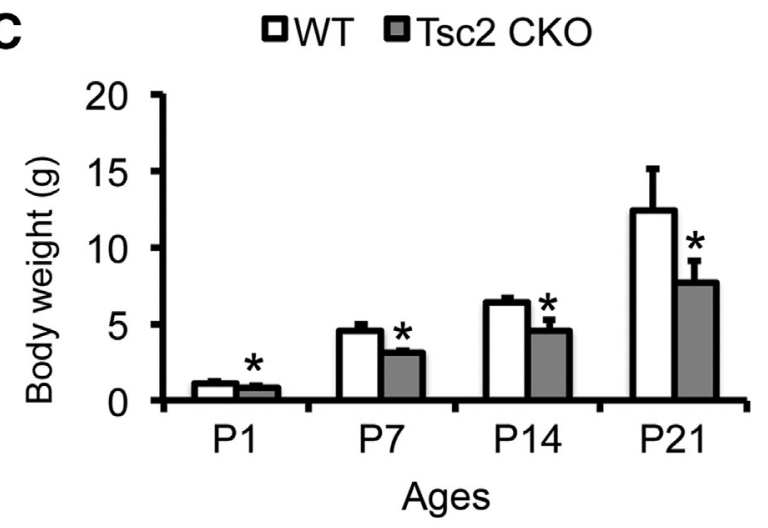

D

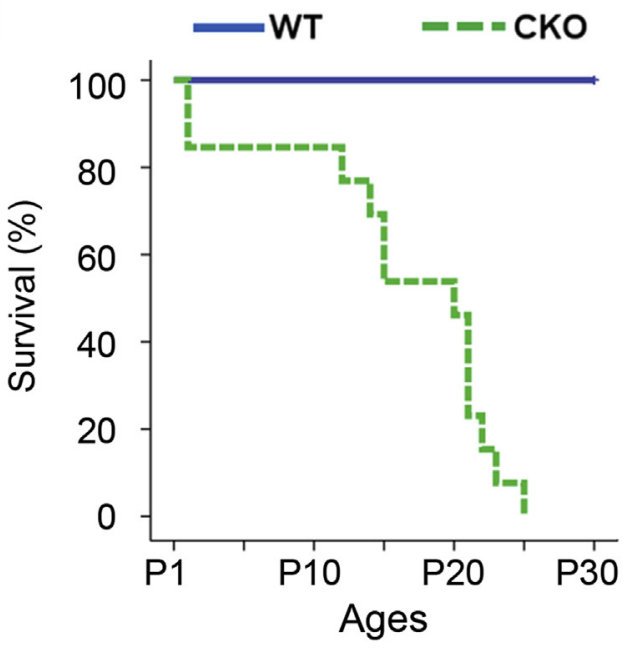

looked pale, polycystic, and enlarged by gross view (Figure 3D). We then analyzed the dynamic changes of kidney morphology in the Tsc2 CKO mice. Interestingly, no significant alterations of kidney structure and size were observed in neonatal Tsc2 CKO mice at P1 (Figure 4, A and C). Microscopic cystic pathology was observed in the renal cortex from the second week after birth (P14) (Figure 4). The cystic lesions rapidly progressed and expanded into the medulla, destroying the normal renal cortex-medulla structural relationships at 3 weeks of age in all Tsc2 CKO mice (Figure 4). Glycogen accumulation was detected in the cysts by periodic acid-Schiff staining. In addition, multiple foci of tubular epithelial hyperplasia and adenomatous changes adjacent to the cysts were seen in $T s c 2$ CKO kidney after 2 weeks of age (Figure 4, B and C). Consistently, overall cell proliferation in Tsc2 CKO kidneys was doubled compared to that in WT littermates, shown by nucleotide analogue EdU labeling $(9.0 \pm 2.4$ in $T s c 2$ CKO versus $4.1 \pm 1.1$ in WT) (Figure $5, \mathrm{~A}$ and $\mathrm{B}$ ) at P7 before obvious morphological changes occurred. The cystic changes were further analyzed by staining the tissue with L. tetragonobolus lectin (a proximal tubular marker) and D. biflorus agglutinin (a collecting duct marker). Interestingly, some of the lining epithelial cells within the cystic structures were positive for D. biflorus agglutinin (Figure 5C), but not for L. tetragonobolus lectin, which suggests that the cystic pathology originates in the distal collecting ducts. The renal endothelial networks were comparable between Tsc2 CKO and WT controls (Figure 5C). Some of the tubular epithelial cells were positively stained with vimentin in the $T s c 2$ CKO kidney at P21, with significant reduction of Cdh1 expression (Figure 5D). Increased vimentin protein level in the Tsc2 CKO kidney was verified by Western blot (Figure 5E), suggesting an epithelialmesenchymal transition as a consequence of Tsc2 inactivation. In addition, increased apoptotic cells, detected by active caspase 3 immunostaining, were seen in P21 Tsc2 CKO kidney sections (Figure 5, F and G). These apoptotic cells in the cysts appeared to be shed into the lumens. Interestingly, only minor increase of serum cystatin $\mathrm{C}$ was detected in the live $T s c 2 \mathrm{CKO}$ mice around the age of $\mathrm{P} 20(10 \pm 3 \%$ greater than the littermate WT control; ${ }^{*} P<0.05, n=5$ pairs), suggesting that kidney function is not severely impaired.

\section{Mesodermal Tsc2 Deletion Causes Retardation of Pulmonary Alveolar Development}

Using mT-mG fluorescence reporter, we confirmed that most lung mesenchymal cells, except pulmonary endothelial

Figure 1 Deletion of $T s c 2$ in mesoderm-derived cells resulted in reduced body growth and lethality at early life. A: LacZ staining (blue) of embryonic day 14.5 Dermo1-Cre/Gt(ROSA)26Sor ${ }^{\text {tm1Sor }}$ reporter mice to visualize the pattern of Cre expression. B: Comparison of body sizes between $T s c 2^{\mathrm{fx} / \mathrm{fx}} /$ Dermo1-Cre [Tsc2 conditional knockout (CKO)] mice and their wild-type (WT) littermate controls. C: Comparison of whole body weights between TsC2 CKO and their littermate controls at different ages. D: Altered survival was detected in Tsc2 CKO mice compared to the WT controls. $n=4$ per genotype (C); $n=13$ (4 females and 9 males; D). ${ }^{*} P<0.05$. $P$, postnatal day. 


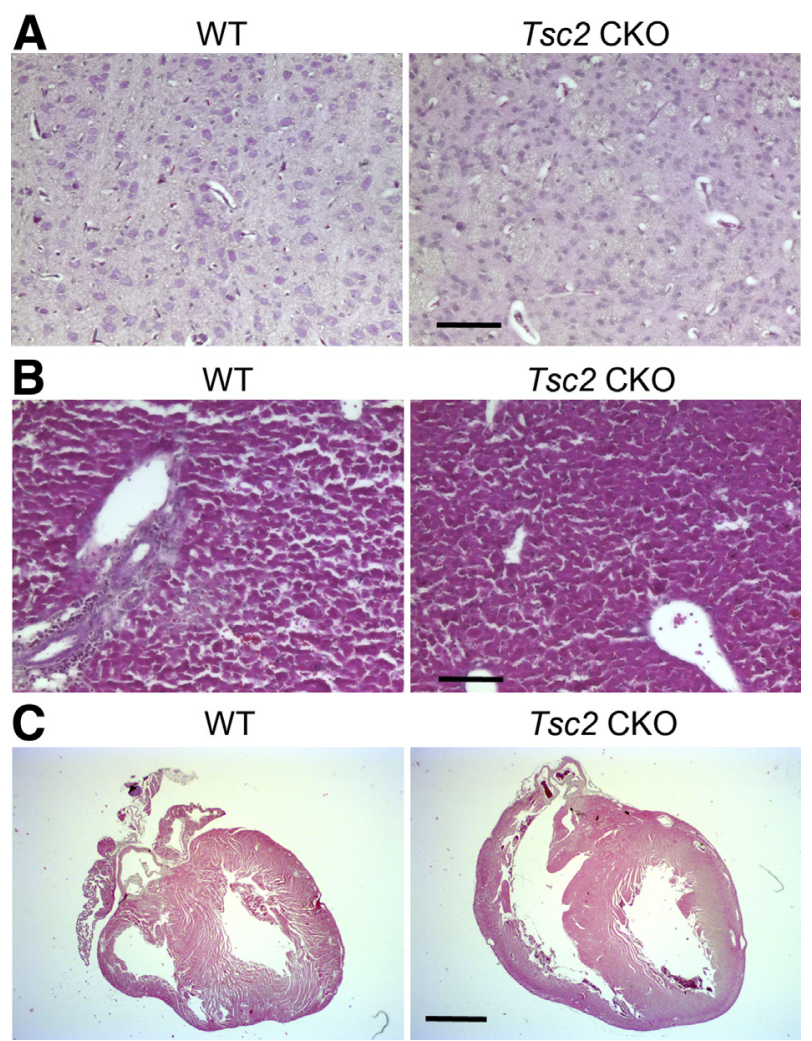

Figure 2 Histological comparison of brain (A), liver (B), and heart (C) between $T s c 2^{\mathrm{fx} / \mathrm{fx}} /$ Dermo1-Cre female mice and wild-type (WT) female control mice at postnatal day 14 is shown by hematoxylin and eosin-stained tissue sections. Scale bars: $100 \mu \mathrm{m}$ (A and B); $1 \mathrm{~mm}$ (C). CKO, conditional knockout.

cells, were targeted by the Dermo1-Cre driver during development, whereas no epithelial cells were Cre positive (Figure 6, A and B). The deletion of $T s c 2$ in the lung tissue of Dermo1-Cre/Tsc2 $2^{\mathrm{fx} / \mathrm{fx}}$ mice was then validated by reduced Tsc2 protein expression and increased mTOR pathway activity (phosphorylation of S6) using Western blot and immunostaining (Figure 6, C and D). Lung development was examined by comparing morphological structures between $T s c 2$ CKO mice and their WT littermate controls. Although prenatal development of the lung in $T s c 2$ CKO mice was not significantly affected, shown by their lung morphology at P1, significant deficiency of lung alveolarization, which normally occurs from P5 to P30 in mice, ${ }^{24}$ was detected (Figure 6E). In the Tsc2 CKO mouse lung, growth of alveolar septa was markedly decreased, which resulted in larger alveolar spaces, within the lungs from P7 to P21. This altered histology was then verified by morphometric measurement of average alveolar size (mean linear intercept) (Figure 6F). Therefore, lung mesenchymal Tsc2 has an important functional role in promoting lung alveolarization during postnatal development.

To understand the potential mechanisms by which mesenchymal $T s c 2$ deletion inhibited lung alveolarization, we analyzed cell proliferation and differentiation. Interestingly, a significant reduction of cell proliferation was detected in P7 Tsc2 CKO mouse lungs using nuclear EdU labeling $(6.7 \pm 1.2 \%$ in $T s c 2$ CKOs versus $11.0 \pm 1.8 \%$ in WT controls) (Figure 7, A and B). Differentiation analysis using immunostaining revealed that lung alveolar myofibroblasts in the $T s c 2$ CKO mice were markedly reduced, as shown by $\alpha$-smooth muscle actin-positive cells in alveolar septa (Figure 7C), whereas smooth muscle cells of both airways and vasculatures appeared similar between $T s c 2$ $\mathrm{CKO}$ and the controls. Interestingly, type II alveolar epithelial cells, shown by Sp-C staining, were also significantly decreased, whereas type I alveolar epithelial cells, shown by T1 $\alpha$ staining, were not changed (Figure 7C). The proximal airway epithelial cells, including ciliated cells and Club cells, shown by $\beta$-tubulin IV and CC10 immunostaining, respectively, were comparable between $T s c 2$ CKO and WT mice. The number and distribution of alveolar capillary endothelial cells, detected by platelet endothelial cell adhesion molecule 1 staining, appeared to be normal (Figure 7C). Finally, elastin fiber deposition in the tips of alveolar septa and alveolar walls was attenuated in the $T s c 2$ CKO lung during alveolar stage (P14) (Figure 7, D and E). Therefore, reduced lung alveolarization in lung mesenchyme-specific $T s c 2$ knockout mice is likely caused by both altered cell proliferation and myofibroblast differentiation in the peripheral lung.

\section{Discussion}

TSC is a disease affecting multiple organs and systems. Germline homozygous deletion of $T s c 2$ in mice results in embryonic lethality, and $T s c 2$ heterozygous deletion results in liver, kidney, and lung tumors as the primary phenotypes in adult mice. ${ }^{7-9}$ The manifestations of human TSC include mesenchymal-lineage tumors of the kidney (angiomyolipomas) and lung (lymphangioleiomyomatosis or LAM). To elucidate the consequences of TSC2 inactivation in multiple mesenchymal lineages, we used the Dermol-promoter-Cre that drives Cre expression in mesoderm-derived cells of multiple organs. ${ }^{15}$ Herein, we report the novel findings that pan-mesenchymal Dermol-Cre-driven homozygous Tsc2 CKO mice are viable but have growth retardation, postnatal progressive polycystic kidney disease, and defective alveolarization of the lung.

Mesenchymal cells are heterogeneous, including a variety of smooth muscle cells, endothelial cells, fibroblasts, osteoblasts, and chondrocytes. The specific mesenchymal cell types and mechanisms underlying the reduced body size and weight in our $T s c 2$ conditional knockout mice are not known. Increased craniofacial bone mass in neural crest-specific $T s c 1$ knockout mice and increased postnatal skeletal bone acquisition in osteoblast-specific $T s c 2$ knockout mice have been reported previously. ${ }^{25,26}$ However, retardation of body growth (size or weight) was not found in those prior models. The reduced body size in our mesenchymal knockout model may be the result of aberrant bone development because of loss of 


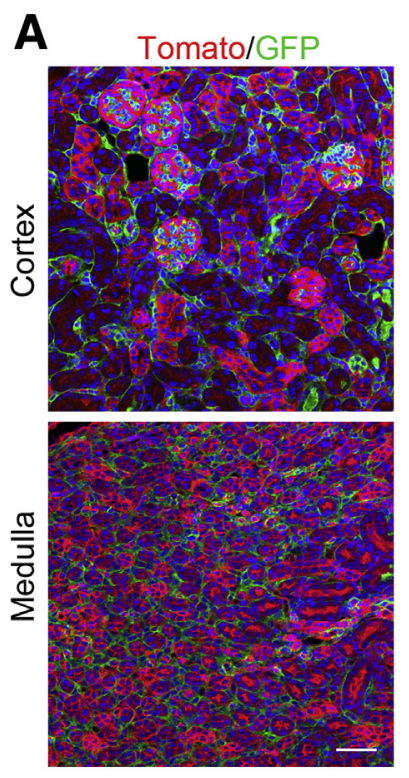

C

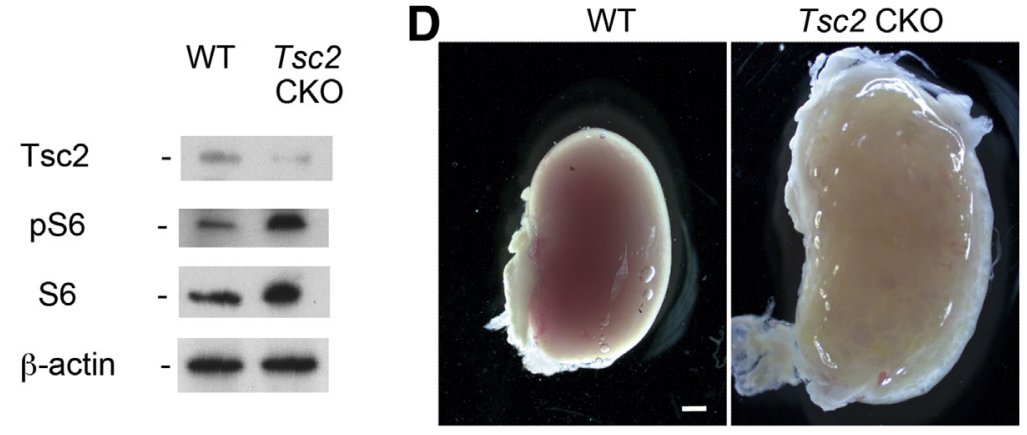

B Cdh1/GFP
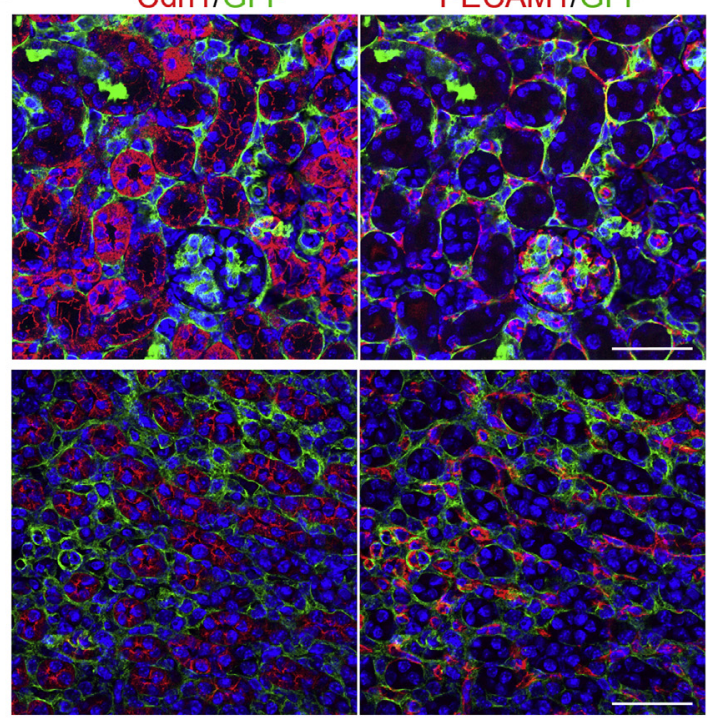

Figure 3 Dermo1-Cre driven Tsc2 deletion in mouse kidney. A: Dermo1-Cre targeted cells in the kidney were characterized in $\mathrm{mT}$-mG fluorescence protein reporter mice at postnatal day 14 (P14), in which membrane green fluorescent protein (GFP) expression (green) replaced membrane Tomato expression (red) only in cells in which Cremediated LoxP DNA recombination occurred. B: Dermo1-Cre-mediated loxP DNA recombination in $\mathrm{mT}-\mathrm{mG}$ reporter mice (green) was not detected in P14 kidney epithelial cells $\left(\mathrm{Cdh}^{+}\right)$or in endothelial cells [platelet endothelial cell adhesion molecule 1 (PECAM1) $^{+}$]. C: Alterations of Tsc2 protein and phosphorylation of $\mathrm{S} 6$, a downstream target of mTOR pathway, were detected in P7 Tsc2 conditional knockout (CKO) kidneys by Western blot. D: Gross view of kidneys isolated from Tsc2 CKO mice and wild-type (WT) control mice at P25. Scale bars: $50 \mu \mathrm{m}$ (A and B); $1 \mathrm{~mm}$ (D).
Tsc2 function in both chrondrocytes and osteoblasts, and/or the consequence of impaired renal or respiratory function. In our Dermo1-Cre mice, Cre is expressed in condensed mesenchyme from which both chondrocytes and osteoblasts are derived during embryonic endochondral ossification (E11.5). ${ }^{15}$ At later stages, except in bone marrow cells and osteoclasts, Cre expression in Dermo1-Cre mice is also detected in chondrocytes of growth plate cartilage and osteoblasts of the perichondrium, periosteum, and endosteum. ${ }^{15} \mathrm{~A}$ future detailed analysis of Tsc2 in these targeted cells and skeletal development will be needed to understand the relevant mechanisms underlying the phenotype of retarded body growth.

Previous studies found that deletion of Tscl in smooth muscle cells and cardiomyocytes using an SM22-driven Cre resulted in severe biventricular hypertrophy starting from embryonic day 15, with median survival of these Tscl conditional knockout mice of approximately 24 days after birth. ${ }^{12,13}$ Interestingly, modest cystadenoma pathology was also detected in the kidney and thought to be caused by minor leakage of Cre expression into renal epithelial cells. In the lung, no significant changes in alveolarization were reported; instead, significant muscularization of small peripheral pulmonary vessels was noted. In our Dermol-Cre-driven $T s c 2$ knockout mice, no morphological change in heart development was detected (Figure 2), which may be because of lack of cardiomyocyte targeting in our Dermol-Cre driver line (Figure 1). However, in the kidney, our Tsc2 CKO mice developed severe polycystic pathology within 3 weeks after birth, resulting in destruction of normal kidney structure. In addition, tubular epithelial hyperplasia and epithelialmesenchymal transition, but no angiomyolipoma-like lesions, appeared by 3 weeks of age. Using the fluorescence reporter to track the Dermol-Cre targeted cells, we found that Cre was expressed specifically in kidney mesenchymal cells, and not in glomerular endothelial cells nor tubular epithelial cells (Figure 3). In addition, overall cell proliferation was increased in the Tsc2 CKO kidney, consistent with epithelial hyperplasia. The potential mechanisms through which mesenchymal $T s c 2$ deletion results in tubular epithelial hyperplasia and cystic change may include a physical blockage of flow by these hyperplastic tubular structures. These findings suggest that Tsc2 functions within renal mesenchymal cells to maintain normal renal tubular structural homeostasis after development rather than regulating kidney morphogenesis per se.

Kidney lesions occur in most TSC patients and are a common cause of morbidity. These include both 
A

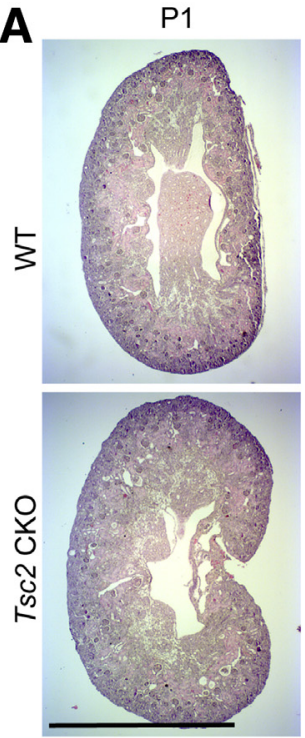

B

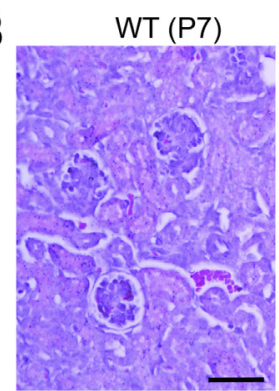

C
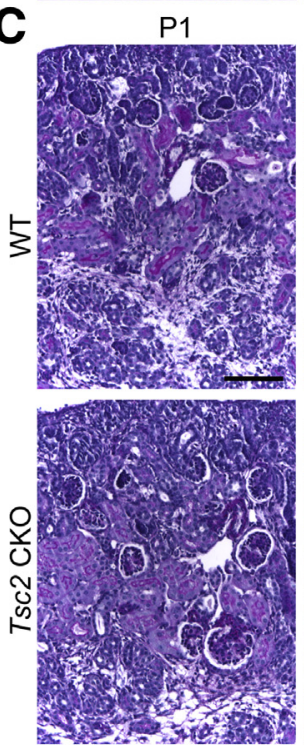

P7

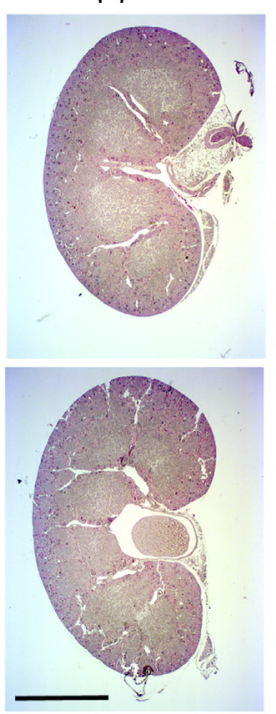

Tsc2 CKO (P7)

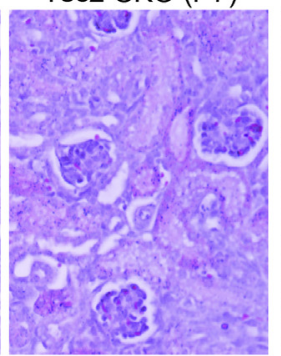

P7
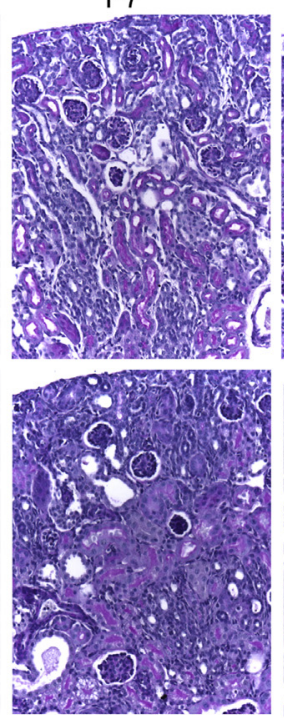

P14

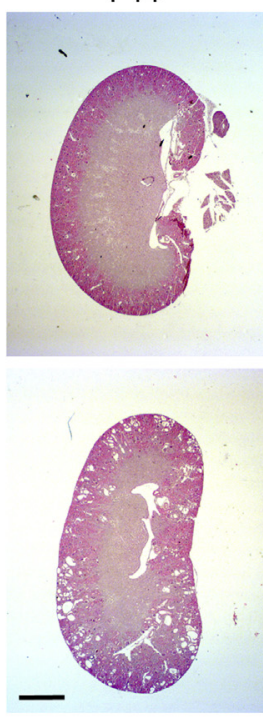

WT (P14)

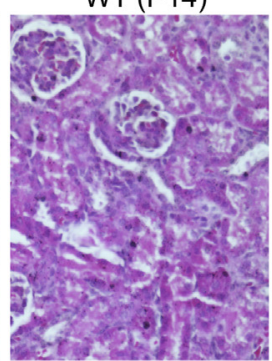

P14
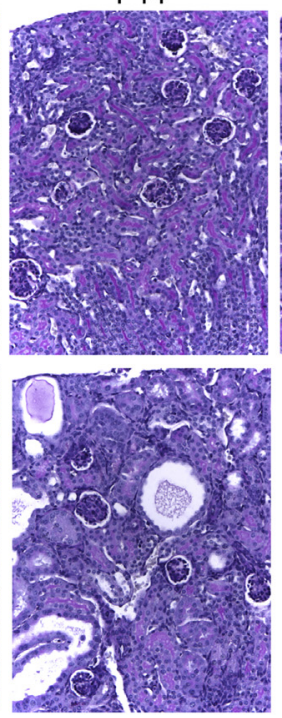

P25
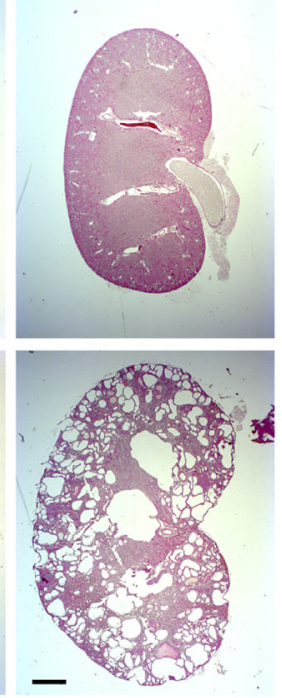

Tsc2 CKO (P14)

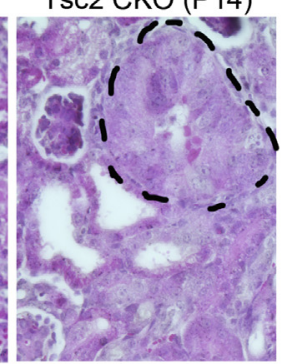

$\mathrm{P} 25$
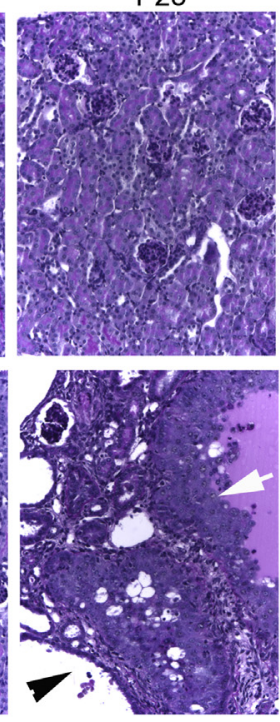

Figure 4 Renal pathology in the Tsc2 conditional knockout (CKO) mice. A: Comparison of overall kidney anatomical structures between the TsC2 CKO mice and their littermate controls at different postnatal ages. B: Hematoxylin and eosin-stained kidney tissue sections at high magnification. Black dotted line highlights a hyperplastic tubule. C: Kidney cortex histology shown by periodic acid-Schiff staining. Arrow indicates multiple layers of hyperplastic tubular epithelia lining a cystic structure; arrowhead, a single layer of epithelium lining a cystic structure. Scale bars: $1 \mathrm{~mm}$ (A); $50 \mu \mathrm{m}$ (B); $100 \mu \mathrm{m}$ (C). P, postnatal day. mesenchymal and epithelial lesions: mesenchymal angiomyolipomas and epithelial polycystic kidney disease, oncocytoma, and renal cell carcinoma. ${ }^{27,28}$ The mechanisms of renal cystic disease in TSC, including whether they arise because of epithelial versus mesenchymal inactivation of TSC2, are not yet completely understood. Several findings have focused on an epithelial pathogenesis model, including that TSC2 is required for the membrane localization of polycystin 1 protein and that TSC2 physically interacts with polycystin 1 to regulate the primary cilium in epithelia. ${ }^{29,30}$ Our data suggest, for the first time, that some of the epithelial cystic lesions in TSC may arise through mesenchymal loss of TSC2 function, supporting a new model for the cyst pathogenesis in TSC. A contiguous gene syndrome, with severe early-onset cystic disease, occurs in human with germline deletions of both TSC2 and 

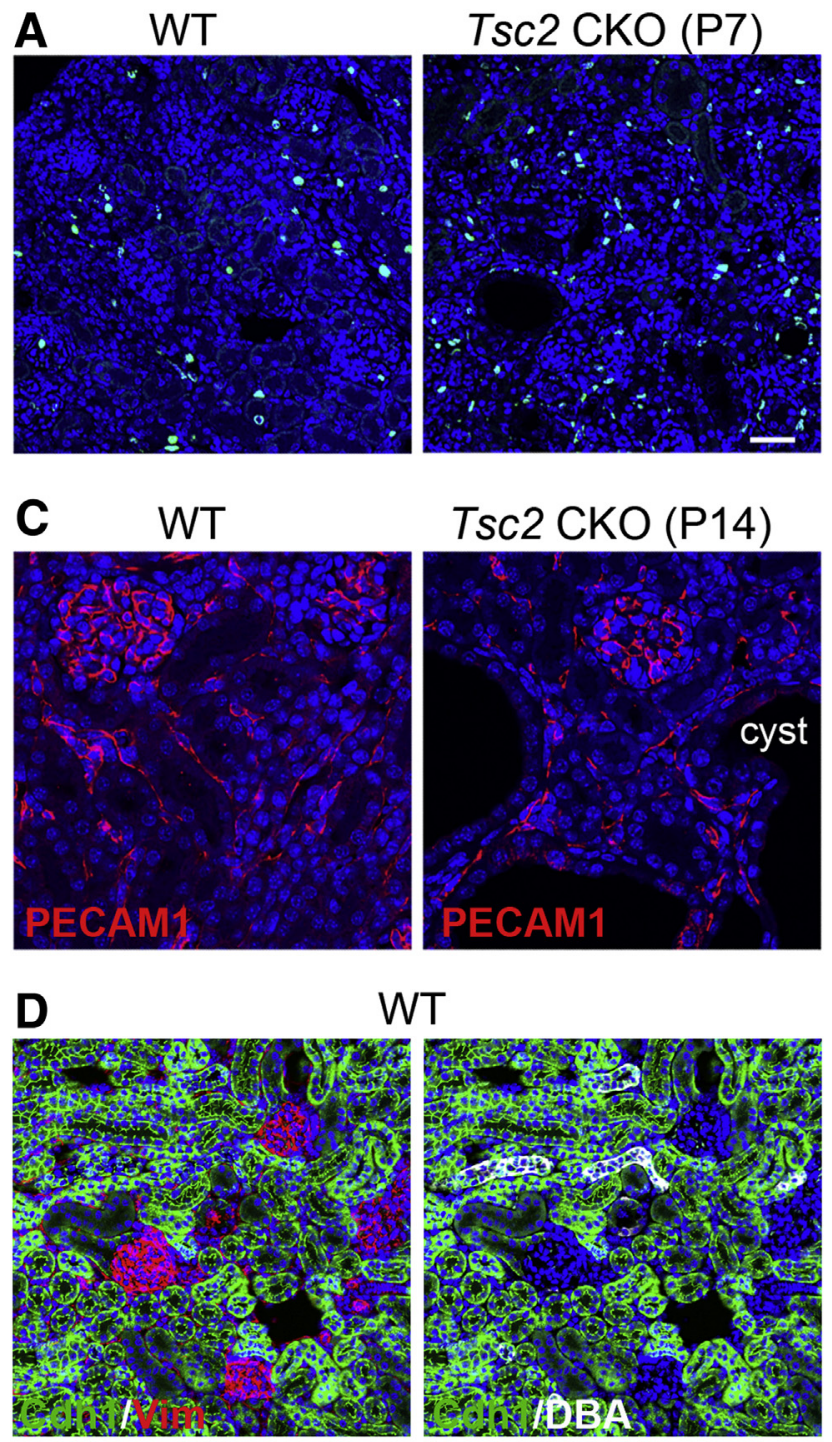

E

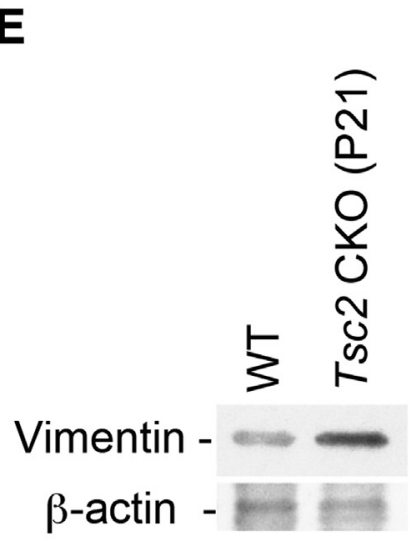

WT

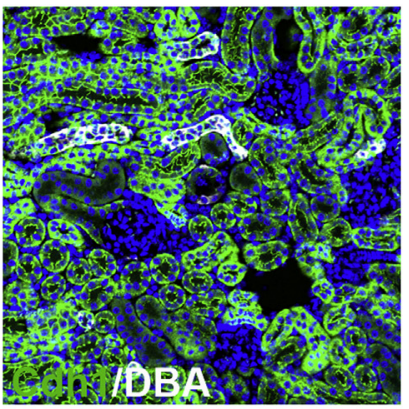

F

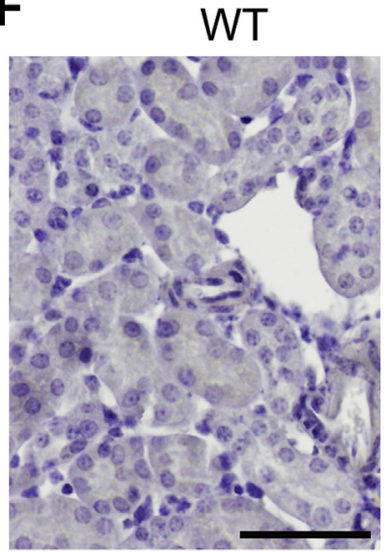

B
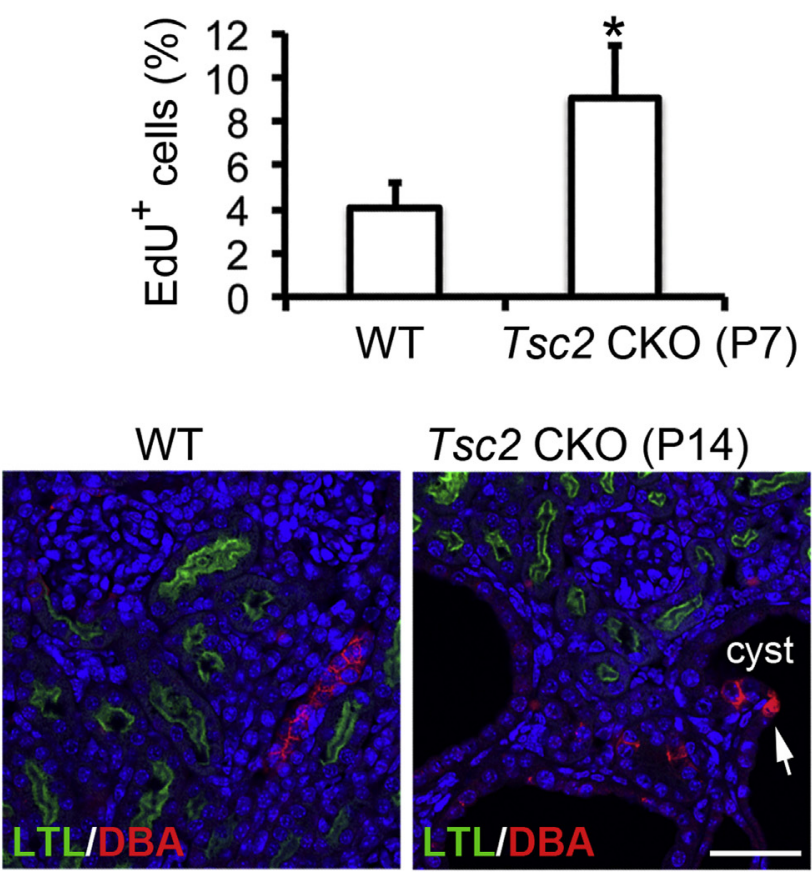

Tsc2 CKO (P21)
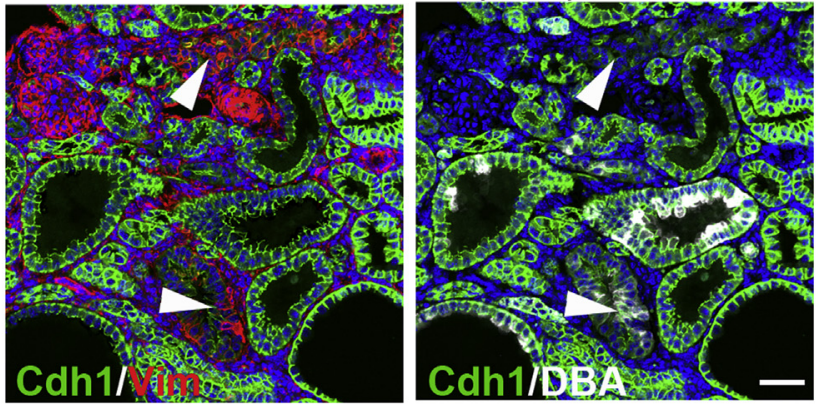

Tsc2 CKO (P21)

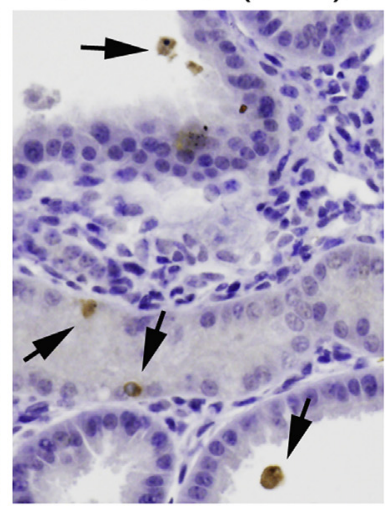

G

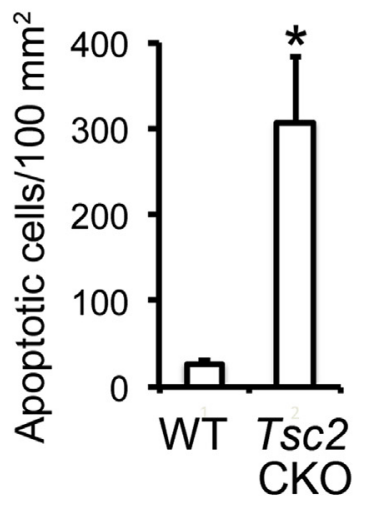

Figure 5 Cell proliferation and cystic epithelial cells in Tsc2 conditional knockout (CKO) kidney. A and B: Proliferative cells were detected by 3-hour 5'-ethynyl2'-deoxyuridine (EdU) labeling, and the percentage of EdU-positive nuclei (green) was analyzed based on DAPI-nuclear counterstaining (blue). C: Endothelial cells in the renal cortex were detected by platelet endothelial cell adhesion molecule 1 (PECAM1) immunostaining. Different tubular epithelial cells were also labeled with Lotus tetragonobolus lectin (LTL; green) or Dolichos biflorus agglutinin (DBA; red)-specific binding. DBA-positive cells in the cystic epithelia are indicated with an arrow. D: Coimmunostaining of Cdh1 (green), vimentin (red), and DBA (gray) for postnatal day 21 (P21) kidneys with indicated Tsc2 genotypes. Vimentinpositive cells in tubules are indicated with arrowheads. E: Increased vimentin in total kidney tissue lysate at P21 was detected by Western blot. $\beta$-Actin is a loading control. F: Activated caspase 3 immunostaining for apoptotic cells (brown; arrows) in P21 kidneys. G: The numbers of apoptotic cells per $100 \mathrm{~mm}^{2}$ area of kidney tissue sections are compared. $n=3$ in each genotype (B and $\mathbf{G}) .{ }^{*} P<0.05$. Scale bars $=50 \mu \mathrm{m}(\mathbf{A}, \mathbf{C}, \mathbf{D}$, and $\mathbf{F})$. WT, wild type. 

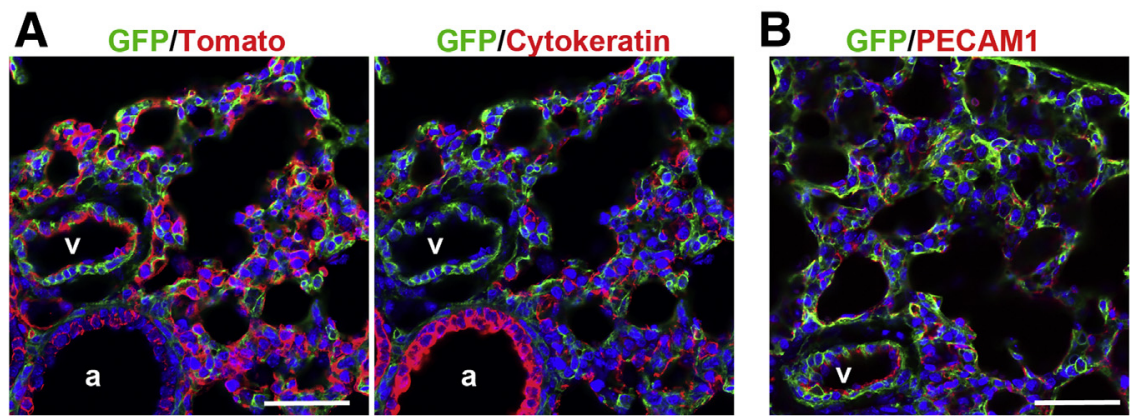

C

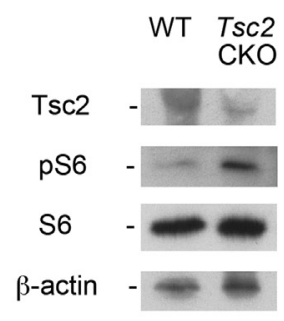

D
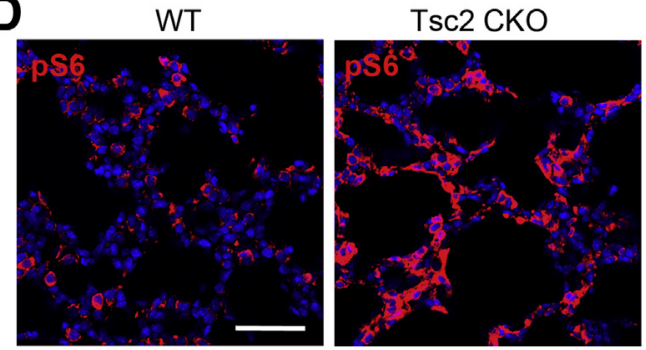

E P1
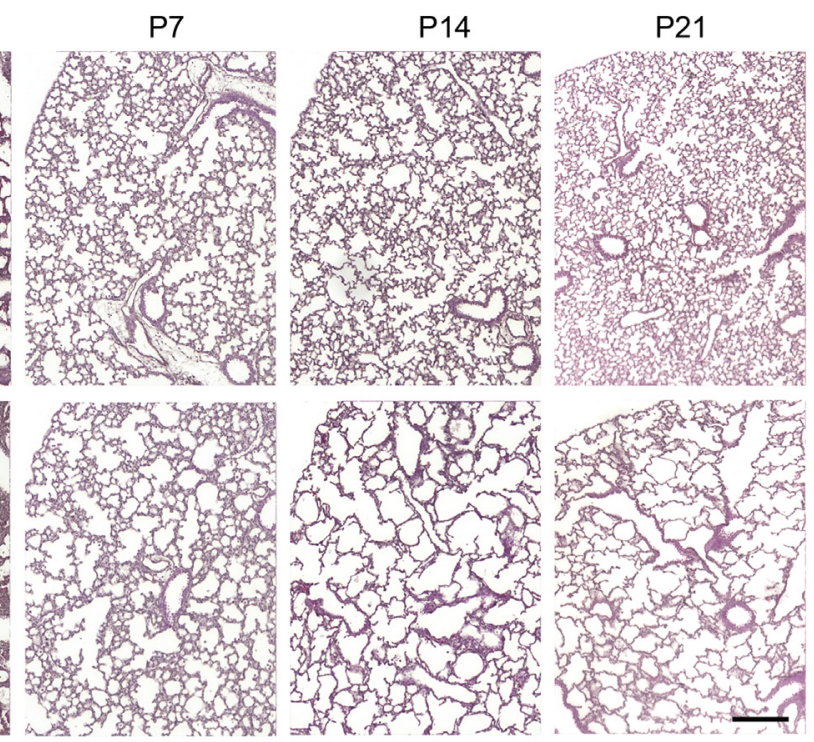

$\mathbf{F}$

DWT $\mathbf{D T s} 2 \mathrm{CKO}$

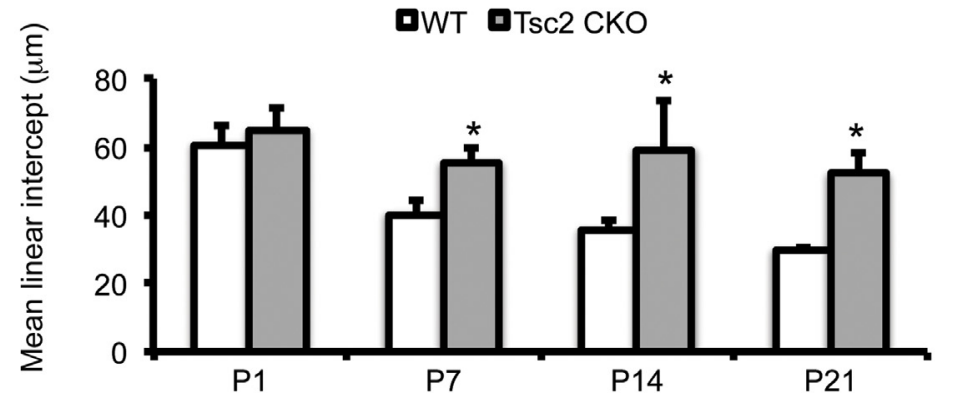

the adjacent polycystic kidney disease 1 (PKDl) gene. ${ }^{31}$ More important, specific deletion of $T s c 2$ exon 2 to 4 in our model confirms that polycystic renal pathology can be caused by mesenchymal loss of $T s c 2,{ }^{16}$ without simultaneous deletion of the adjacent $P k d l$ gene, ${ }^{32}$ which experimentally supports clinical observations that renal cysts can occur in patients with TSC1 or TSC2 mutation but no PKD1 deletion. ${ }^{3}$

In our $T s c 2$ CKO mouse lung, a striking reduction of pulmonary alveolarization was observed, without obvious changes in pulmonary vascular and airway smooth muscle cells. Yet, by examining the alveolar septa structure, 
A

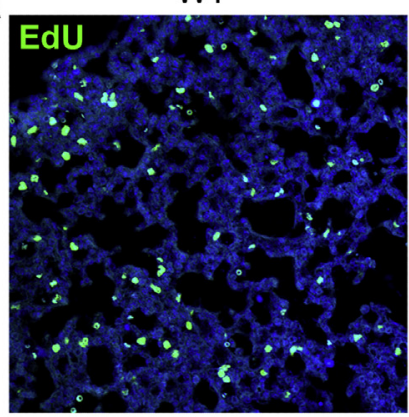

C
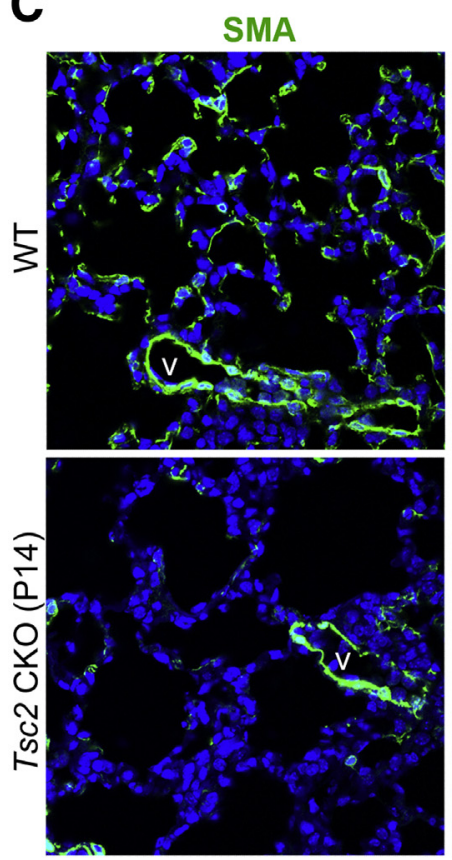

D $\quad W T(P 7)$

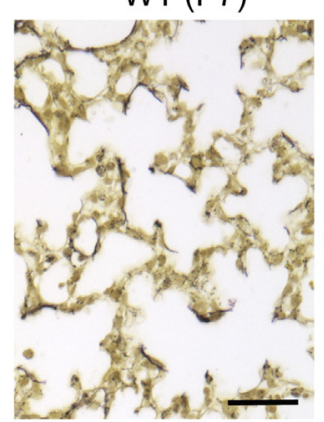

Tsc2 CKO lung (P7)

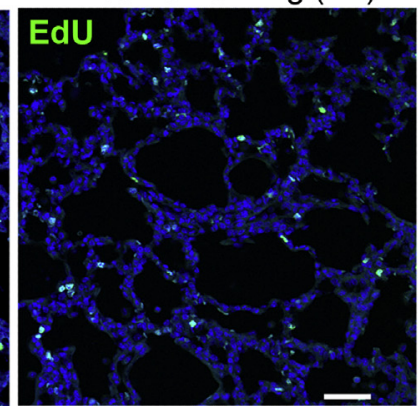

PECAM1
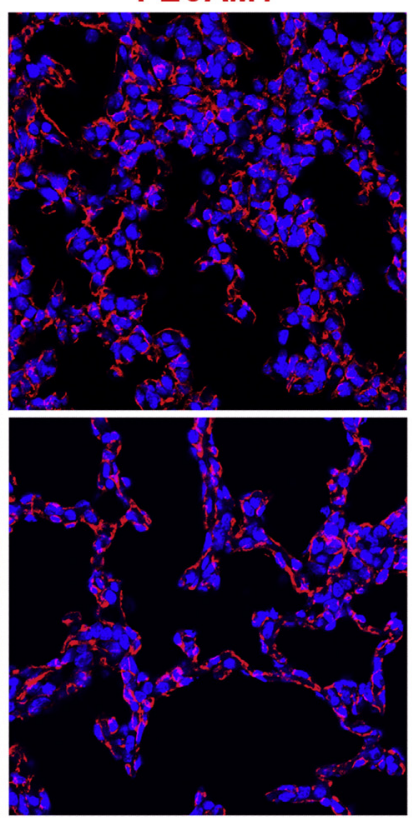

Tsc2 CKO (P7)

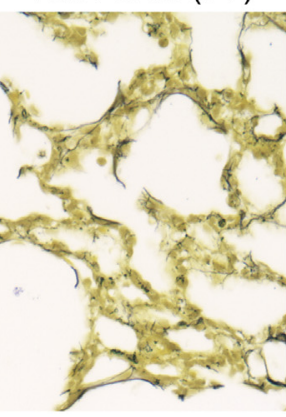

WT (P14)

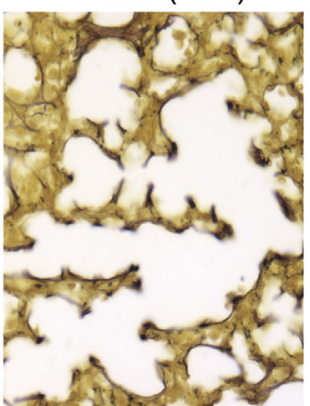

B

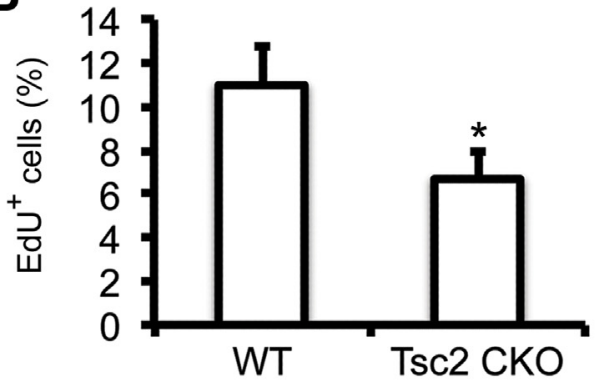

Figure 7 Altered lung proliferation and differentiation in Tsc2 conditional knockout (CKO) mice. A and B: Proliferative cells were detected by 3-hour 5'-ethynyl-2'-deoxyuridine (EdU) labeling (green) in postnatal day 7 (P7) mice, and quantified based on cell nuclear counterstaining with DAPI (blue). C: Lung myofibroblasts/smooth muscle cells, endothelial cells, type II and type I alveolar epithelial cells, airway club cells, and ciliated cells were immunostained with the related protein markers. D: Comparison of elastin fibers (black) in lung tissues between Tsc2 CKO and wild-type (WT) control mice at P7 and P14. E: Semiquantification of elastin deposition in P14 lung tissues, measured by the percentage of stained elastin area over total lung tissue area of four tissue sections. ${ }^{*} P<0.05$. Scale bars $=50 \mu \mathrm{m}(\mathbf{A}-\mathbf{D})$. a, airway; PECAM1, platelet endothelial cell adhesion molecule $1 ;$ SMA, $\alpha$-smooth muscle actin; $v$, vasculature.

we found that $\alpha$-smooth muscle actin-positive myofibroblasts in our Tsc2 CKO lung were significantly reduced, particularly in the tips of the alveolar septa. In addition, overall lung cell proliferation was significantly decreased in the Tsc2 CKO lung, which is opposite to the increased cell proliferation detected in the $T s c 2 \mathrm{CKO}$ kidney. Therefore, it appears that loss-of-function mutation in $T s c 2$ and subsequent mTORC1 hyperactivation result in strikingly diverse cellular responses depending on cell lineages, organs, and stages of development and 
homeostasis. Lung alveolarization occurs postnatally in mice, with the growth of secondary alveolar septa into primary alveolar sacs. Alveolar myofibroblasts play a critical role in driving alveolar septa formation by proliferating and migrating into the tips of the septa and by producing elastin extracellular matrices. ${ }^{34}$ Defects in alveolar myofibroblast migration to the tip because of abrogation of platelet-derived growth factor signaling results in arrest of pulmonary alveolarization. ${ }^{35}$ In our Tsc2 CKO lung, the overall number of alveolar myofibroblasts was significantly reduced, accompanied by decreased elastin deposition within the septa. Therefore, reduced proliferation, differentiation, and function of alveolar myofibroblasts because of loss of Tsc2 function and the resulting hyperactivation of mTORC1 pathway is one of the pivotal mechanisms underlying the alveolar hypoplastic lung phenotype. In contrast, alveolar capillaries were not significantly altered based on immunostaining, suggesting that alveolar angiogenesis, another driving force for pulmonary alveolarization, may not be a critical Tsc2-dependent mechanism for alveolarization. Whether defective alveolarization in these $T s c 2$ CKO mice contributes to pulmonary cystic lesions in adult remains unknown, because of their shortened life span. It has been reported that decreased alveolar growth in early life has a negative impact on alveolar homeostasis in an aging population, associated with alveolar destruction and emphysema. ${ }^{36}$ Therefore, it is possible that reduced alveolarization during lung development may contribute to the pulmonary cystic lesions observed in TSC patients. More important, no LAM-like nodules were detected in the female or male Tsc2 CKO mice, which died before adulthood. We therefore speculate that the high incidence pulmonary cysts in women with TSC, estimated at $80 \%$ by the age of 40 years, ${ }^{37}$ may reflect both a developmental alveolar defect involving TSC1/2 inactivation in the lung mesenchyme that is not sex specific, and sexspecific LAM cell-produced proteases and cytokines that mediate tissue destruction. This model could explain the fact that most TSC patients (both male and female) with lung cysts will not progress to symptomatic pulmonary disease. We further speculate that the mesenchymal impact of TSC2 loss cooperates with LAM cell-produced proteases to enhance the lung destruction. To address these hypotheses, further investigation of the relationship between lung mesenchymal Tsc2 inactivation and LAM-like pathology is needed.

\section{Acknowledgments}

We thank Dr. David M. Ornitz (Washington University, St. Louis, MO) for providing Dermo1-Cre mice; Dr. Michael Gambello (Emory University, Atlanta, GA) for providing floxed-Tsc2 mice; Dr. Esteban Fernandez (Cell Imaging Core of Children's Hospital Los Angeles) for helping with confocal imaging; Dr. Kevin Lemley (Children's Hospital Los Angeles) for initial discussion about mouse kidney phenotype; and Dr. Roderick Bronson (Harvard Medical School) for review of the renal pathology.

\section{References}

1. Curatolo P, Maria BL: Tuberous sclerosis. Handb Clin Neurol 2013, 111:323-331

2. Henske EP, Jóźwiak S, Kingswood JC, Sampson JR, Thiele EA: Tuberous sclerosis complex. Nat Rev Dis Primers 2016, 2:16035

3. Hayashi T, Kumasaka T, Mitani K, Yao T, Suda K, Seyama K: Loss of heterozygosity on tuberous sclerosis complex genes in multifocal micronodular pneumocyte hyperplasia. Mod Pathol 2010, 23: $1251-1260$

4. Carsillo T, Astrinidis A, Henske EP: Mutations in the tuberous sclerosis complex gene TSC2 are a cause of sporadic pulmonary lymphangioleiomyomatosis. Proc Natl Acad Sci U S A 2000, 97: 6085-6090

5. Henske EP, McCormack FX: Lymphangioleiomyomatosis: a wolf in sheep's clothing. J Clin Invest 2012, 122:3807-3816

6. Harari S, Torre O, Moss J: Lymphangioleiomyomatosis: what do we know and what are we looking for? Eur Respir Rev 2011, 20:34-44

7. Kwiatkowski DJ, Zhang H, Bandura JL, Heiberger KM, Glogauer M, elHashemite N, Onda H: A mouse model of TSC1 reveals sex-dependent lethality from liver hemangiomas, and up-regulation of p70S6 kinase activity in Tsc1 null cells. Hum Mol Genet 2002, 11:525-534

8. Kobayashi T, Minowa O, Kuno J, Mitani H, Hino O, Noda T: Renal carcinogenesis, hepatic hemangiomatosis, and embryonic lethality caused by a germ-line Tsc 2 mutation in mice. Cancer Res 1999, 59:1206-1211

9. Onda H, Lueck A, Marks PW, Warren HB, Kwiatkowski DJ: Tsc2(+/-) mice develop tumors in multiple sites that express gelsolin and are influenced by genetic background. J Clin Invest 1999, 104:687-695

10. Crowell B, Hwa Lee G, Nikolaeva I, Dal Pozzo V, D’Arcangelo G: Complex neurological phenotype in mutant mice lacking Tsc2 in excitatory neurons of the developing forebrain(123). eNeuro 2015, 2: ENEURO.0046-15.2015

11. Ma A, Wang L, Gao Y, Chang Z, Peng H, Zeng N, Gui YS, Tian X, Li X, Cai B, Zhang H, Xu KF: Tsc1 deficiency-mediated mTOR hyperactivation in vascular endothelial cells causes angiogenesis defects and embryonic lethality. Hum Mol Genet 2014, 23:693-705

12. Kayyali US, Larsen CG, Bashiruddin S, Lewandowski SL, Trivedi CM, Warburton RR, Parkhitko AA, Morrison TA, Henske EP, Chekaluk Y, Kwiatkowski DJ, Finlay GA: Targeted deletion of Tsc1 causes fatal cardiomyocyte hyperplasia independently of afterload. Cardiovasc Pathol 2015, 24:80-93

13. Malhowski AJ, Hira H, Bashiruddin S, Warburton R, Goto J, Robert B, Kwiatkowski DJ, Finlay GA: Smooth muscle protein-22-mediated deletion of Tsc1 results in cardiac hypertrophy that is mTORC1-mediated and reversed by rapamycin. Hum Mol Genet 2011, 20:1290-1305

14. Kwiatkowski DJ: Animal models of lymphangioleiomyomatosis (LAM) and tuberous sclerosis complex (TSC). Lymphat Res Biol 2010, 8:51-57

15. Yu K, Xu J, Liu Z, Sosic D, Shao J, Olson EN, Towler DA, Ornitz DM: Conditional inactivation of FGF receptor 2 reveals an essential role for FGF signaling in the regulation of osteoblast function and bone growth. Development 2003, 130:3063-3074

16. Hernandez O, Way S, McKenna J, Gambello MJ: Generation of a conditional disruption of the Tsc2 gene. Genesis 2007, 45:101-106

17. Muzumdar MD, Tasic B, Miyamichi K, Li L, Luo L: A global doublefluorescent Cre reporter mouse. Genesis 2007, 45:593-605

18. Soriano P: Generalized lacZ expression with the ROSA26 Cre reporter strain. Nat Genet 1999, 21:70-71

19. Chen H, Zhuang F, Liu YH, Xu B, Del Moral P, Deng W, Chai Y, Kolb M, Gauldie J, Warburton D, Moses HL, Shi W: TGF-beta 
receptor II in epithelia versus mesenchyme plays distinct roles in the developing lung. Eur Respir J 2008, 32:285-295

20. Sun J, Zhuang FF, Mullersman JE, Chen H, Robertson EJ, Warburton D, Liu YH, Shi W: BMP4 activation and secretion are negatively regulated by an intracellular gremlin-BMP4 interaction. J Biol Chem 2006, 281:29349-29356

21. Luo Y, El Agha E, Turcatel G, Chen H, Chiu J, Warburton D, Bellusci S, Qian BP, Menke DB, Shi W: Mesenchymal adenomatous polyposis coli plays critical and diverse roles in regulating lung development. BMC Biol 2015, 13:42

22. Pajak L, Jin F, Xiao GH, Soonpaa MH, Field LJ, Yeung RS: Sustained cardiomyocyte DNA synthesis in whole embryo cultures lacking the TSC2 gene product. Am J Physiol 1997, 273:H1619-H1627

23. Rennebeck G, Kleymenova EV, Anderson R, Yeung RS, Artzt K, Walker CL: Loss of function of the tuberous sclerosis 2 tumor suppressor gene results in embryonic lethality characterized by disrupted neuroepithelial growth and development. Proc Natl Acad Sci U S A 1998, 95:15629-15634

24. Warburton D, Bellusci S, Del Moral PM, Kaartinen V, Lee M, Tefft D, Shi W: Growth factor signaling in lung morphogenetic centers: automaticity, stereotypy and symmetry. Respir Res 2003, 4:5

25. Fang F, Sun S, Wang L, Guan JL, Giovannini M, Zhu Y, Liu F: Neural crest-specific TSC1 deletion in mice leads to sclerotic craniofacial bone lesion. J Bone Miner Res 2015, 30:1195-1205

26. Riddle RC, Frey JL, Tomlinson RE, Ferron M, Li Y, DiGirolamo DJ, Faugere MC, Hussain MA, Karsenty G, Clemens TL: Tsc2 is a molecular checkpoint controlling osteoblast development and glucose homeostasis. Mol Cell Biol 2014, 34:1850-1862

27. Henske EP: Tuberous sclerosis and the kidney: from mesenchyme to epithelium, and beyond. Pediatr Nephrol 2005, 20:854-857

28. Yang P, Cornejo KM, Sadow PM, Cheng L, Wang M, Xiao Y, Jiang Z, Oliva E, Jozwiak S, Nussbaum RL, Feldman AS, Paul E, Thiele EA, Yu JJ, Henske EP, Kwiatkowski DJ, Young RH, Wu CL: Renal cell carcinoma in tuberous sclerosis complex. Am J Surg Pathol 2014, 38:895-909
29. Kleymenova E, Ibraghimov-Beskrovnaya O, Kugoh H, Everitt J, Xu H, Kiguchi K, Landes G, Harris P, Walker C: Tuberin-dependent membrane localization of polycystin-1: a functional link between polycystic kidney disease and the TSC2 tumor suppressor gene. Mol Cell 2001, 7:823-832

30. Shillingford JM, Murcia NS, Larson CH, Low SH, Hedgepeth R, Brown N, Flask CA, Novick AC, Goldfarb DA, Kramer-Zucker A, Walz G, Piontek KB, Germino GG, Weimbs T: The mTOR pathway is regulated by polycystin-1, and its inhibition reverses renal cystogenesis in polycystic kidney disease. Proc Natl Acad Sci U S A 2006, 103:5466-5471

31. Brook-Carter PT, Peral B, Ward CJ, Thompson P, Hughes J, Maheshwar MM, Nellist M, Gamble V, Harris PC, Sampson JR: Deletion of the TSC2 and PKD1 genes associated with severe infantile polycystic kidney disease: a contiguous gene syndrome. Nat Genet 1994, 8:328-332

32. Piontek K, Menezes LF, Garcia-Gonzalez MA, Huso DL, Germino GG: A critical developmental switch defines the kinetics of kidney cyst formation after loss of Pkd1. Nat Med 2007, 13:1490-1495

33. Sampson JR, Maheshwar MM, Aspinwall R, Thompson P, Cheadle JP, Ravine D, Roy S, Haan E, Bernstein J, Harris PC: Renal cystic disease in tuberous sclerosis: role of the polycystic kidney disease 1 gene. Am J Hum Genet 1997, 61:843-851

34. Morrisey EE, Hogan BL: Preparing for the first breath: genetic and cellular mechanisms in lung development. Dev Cell 2010, 18:8-23

35. Bostrom H, Willetts K, Pekny M, Leveen P, Lindahl P, Hedstrand H, Pekna M, Hellstrom M, Gebre-Medhin S, Schalling M, Nilsson M, Kurland S, Tornell J, Heath JK, Betsholtz C: PDGF-A signaling is a critical event in lung alveolar myofibroblast development and alveogenesis. Cell 1996, 85:863-873

36. Fletcher C, Peto R: The natural history of chronic airflow obstruction. Br Med J 1977, 1:1645-1648

37. Cudzilo CJ, Szczesniak RD, Brody AS, Rattan MS, Krueger DA, Bissler JJ, Franz DN, McCormack FX, Young LR: Lymphangioleiomyomatosis screening in women with tuberous sclerosis. Chest 2013, 144:578-585 